\title{
Delayed-onset bronchiolitis obliterans with organising pneumonia associated with massive acetic acid steam inhalation
}

A 34-year-old worker had massive exposure to acetic acid steam from an explosion in a chemical factory. At the emergency department respiratory distress, hoarseness and swelling of the oropharyngeal mucosa were noted. The chest radiograph showed bilateral infiltration (fig 1). After being diagnosed with inhalation injury and chemical pneumonitis, he was prophylactically intubated and given parenteral steroid with prophylactic antibiotics. Diagnostic fibreoptic bronchoscopy revealed diffuse trachobronchial mucosal redness and friability (fig S1A,B available online only). Scattered throughout the airway were multiple corrosive ulcerations. A followup thin-section CT scan at day 15 showed ground-glass attenuation in the bilateral upper lung (fig 2). Lower sections of the chest CT scan did not show signs of severe airway obstruction. At day 21 he

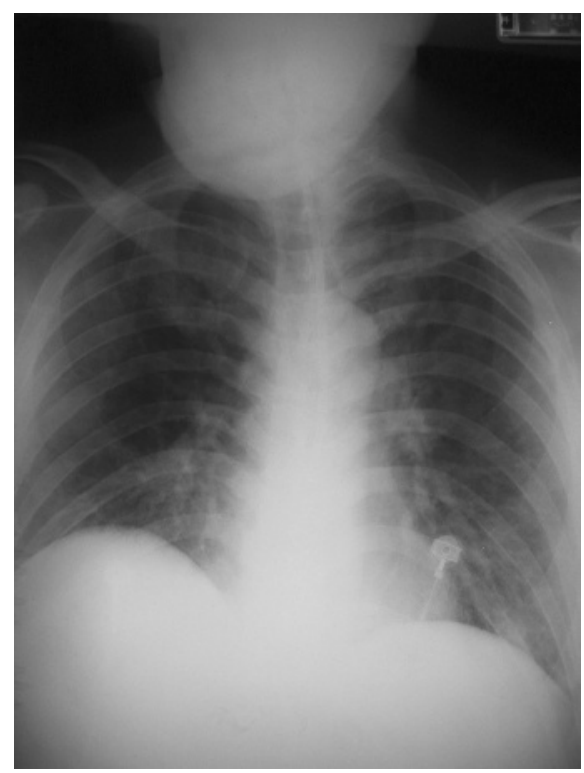

Figure 1 Initial chest radiograph showing bilateral perihilar infiltrates. began to feel chest tightness and dyspnoea and had massive repeated haemoptysis. Repeated bronchoscopy showed diffuse mucosal pallor, scattered ulcerations, fibrous exudates and granulation tissue in the lower trachea, right upper bronchi and more distal bronchioles (fig S1C, D available online only).

Delayed-onset bronchiolitis obliterans organising pneumonia (BOOP) due to acetic acid steam inhalation was diagnosed. He died of an episode of methicillin-resistant Staphylococcus aureus infection of the bloodstream with multiple organ failure 1 month later.

The natural course of pulmonary injury associated with acetic acid steam exposure has not been documented. Our patient experienced a biphasic clinical response characterised by acute pulmonary oedema followed by delayed-onset BOOP, similar to that observed in other
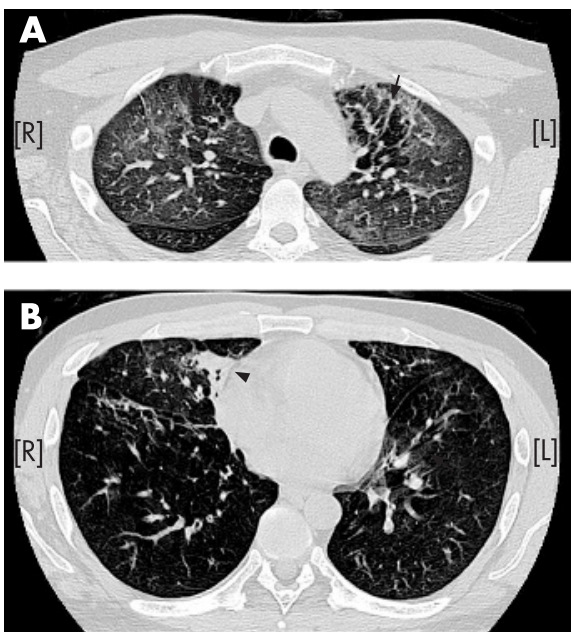

Figure 2 (A) High-resolution CT scan showing diffuse ground-glass attenuation in the bilateral upper lungs. Scattered areas of air trapping are visible (arrows). (B) Lower section showing only scattered peribronchovascular groundglass attenuation (arrow). Patchy consolidation is evident (arrowhead). cases of irritant gas exposure. ${ }^{1}$ The most common symptoms of BOOP are a progressive dry cough, effort dyspnoea, low-grade pyrexia and malaise. Patients with BOOP rarely present with a large amount of haemoptysis and this posed a great diagnostic challenge initially. $^{2}$

\section{B-F Sheu, ${ }^{1}$ C-C Lee, ${ }^{2}$ Y-R Young, ${ }^{1}$ L-F Li, ${ }^{3}$ S-S Chang ${ }^{1,4}$}

${ }^{1}$ Department of Emergency Medicine, Chang Gung Memorial Hospital and Chang Gung College of Medicine Taoyuan, Taiwan; ${ }^{2}$ Department of Emergency Medicine, National Taiwan University Hospital Yun-Lin Branch, Douliou, Taiwan; ${ }^{3}$ Department of Chest Medicine, Chang Gung Memorial Hospital, Taoyuan, Taiwan; ${ }^{4}$ Department of Nursing, Chang Gung Institute of Technology, Taoyuan, Taiwan

Correspondence to: Dr C-C Lee, Department of Emergency Medicine, National Taiwan University Hospital, No. 7 Chung-Shan South Road, Taipei 100,

Taiwan; chnchnglee@yahoo.com

Competing interests: None.

Figure $\mathrm{S} 1$ is published online only at http://thorax.bmj. com/content/vol63/issue6

Thorax 2008;63:570. doi:10.1136/thx.2007.079665

\section{REFERENCES}

Do Pico GA. Toxic gas inhalation. Curr Opin Pulm Med 1995;1:102-8.

2. Epler GR. Bronchiolitis obliterans organizing pneumonia. Arch Intern Med 2001;161:158-64.

\section{Learning points}

- Massive exposure to acetic acid steam may cause a biphasic pulmonary disease, acute pulmonary oedema followed by BOOP, similar to that observed in other cases of irritant gas exposure.

- In patients with irritant gas exposure and compatible radiographic findings, massive haemoptysis is a possible presentation that warrants consideration of BOOP. 